\title{
Bayesian Estimation Based on Rayleigh Progressive Type II Censored Data with Binomial Removals
}

\author{
Reza Azimi $^{1}$ and Farhad Yaghmaei ${ }^{2}$ \\ ${ }^{1}$ Department of Statistics, Parsabad Moghan Branch, Islamic Azad University, Parsabad Moghan, Iran \\ ${ }^{2}$ Faculty of Science, Golestan University, Gorgan, Golestan, Iran \\ Correspondence should be addressed to Reza Azimi; azimireza1365@gmail.com
}

Received 4 October 2012; Revised 27 January 2013; Accepted 3 February 2013

Academic Editor: Mohammad Modarres

Copyright ( 2013 R. Azimi and F. Yaghmaei. This is an open access article distributed under the Creative Commons Attribution License, which permits unrestricted use, distribution, and reproduction in any medium, provided the original work is properly cited.

\begin{abstract}
This study considers the estimation problem for the parameter and reliability function of Rayleigh distribution under progressive type II censoring with random removals, where the number of units removed at each failure time has a binomial distribution. We use the maximum likelihood and Bayesian procedures to obtain the estimators of parameter and reliability function of Rayleigh distribution. We also construct the confidence intervals for the parameter of Rayleigh distribution. Monte Carlo simulation method is used to generate a progressive type II censored data with binomial removals from Rayleigh distribution, and then these data are used to compute the point and interval estimations of the parameter and compare both the methods used with different random schemes.
\end{abstract}

\section{Introduction}

The Rayleigh distribution provides a population model which is useful in several areas of statistics. In the literature, many researcher studied properties of the Rayleigh distribution, particularly in life testing and reliability. Life testing experiments often deal with censored sample in order to estimate the parameters involved in the life distribution. The cumulative distribution function (CDF), probability density function (PDF), and the reliability function of the Rayleigh distribution with parameter $\sigma>0$ are

$$
\begin{gathered}
F(x \mid \sigma)=1-\exp \left\{-\frac{x^{2}}{2 \sigma^{2}}\right\}, \quad x>0, \\
f(x \mid \sigma)=\frac{x}{\sigma^{2}} \exp \left\{-\frac{x^{2}}{2 \sigma^{2}}\right\}, \\
R(x \mid \sigma)=\exp \left\{-\frac{x^{2}}{2 \sigma^{2}}\right\}
\end{gathered}
$$

respectively. Inferences for $\sigma$ in the Rayleigh distribution have been discussed by several authors. Harter and Moore [1] derived an explicit form for the maximum likelihood estimator (MLE) of $\sigma$ based on type II censored data. Moreover, Bayesian estimation and prediction problems for the $\sigma$ based on doubly censored sample have been considered by Fernández [2] and Raqab and Madi [3]. Wu et al. [4] have derived the Bayesian estimator and prediction intervals based on progressively type II censored samples. A recent account on progressive censoring schemes can be obtained in the monograph by Balakrishnan and Aggarwala [5] or in the excellent review article by Balakrishnan [6].

Suppose that $n$ units are placed on a life test and the experimenter decides beforehand quantity $m$, the number of units, to be failed. Now at the time of the first failure, $R_{1}$ of the remaining $n-1$ surviving units are randomly removed from the experiment. Continuing, at the time of the second failure, $R_{2}$ of the remaining $n-R_{1}-2$ units are randomly drawn from the experiment. Finally, at the time of the $m$ th failure, all the remaining $R_{m}=n-m-R_{1}-R_{2}-\cdots-$ 
TABLE 1: A schematic representation of the progressive type II censoring with binomial removals.

\begin{tabular}{lcccc}
\hline Process & The number in life testing & Failures & Binomial removals & Remains \\
\hline 1 & $n$ & 1 & $R_{1} \sim B(n-m, p)$ & $n-1-R_{1}$ \\
2 & $n-1-R_{1}$ & 1 & $R_{2} \sim B\left(n-m-R_{1}, p\right)$ & $n-2-R_{1}-R_{2}$ \\
$\ldots$ & $\ldots$ & $\ldots$ & $\ldots$ & $\ldots$ \\
$m-1$ & $n-(m-2)-\sum_{j-1}^{m-2} R_{j}$ & 1 & $R_{m-1} \sim B\left(n-m-\sum_{j=1}^{m-2} R_{j}, p\right)$ & $n-(m-1)-\sum_{j=1}^{m-1} R_{j}$ \\
$m$ & $n-(m-1)-\sum_{j=1}^{m-1} R_{j}$ & 1 & $R_{m}=n-m-\sum_{j=1}^{m-1} R_{j}$ & 0 \\
\hline
\end{tabular}

$R_{m-1}$ surviving units are removed from the experiment. Note that, in this scheme, $R_{1}, R_{2}, \ldots, R_{m}$ are all prefixed. However, in some practical situations, these numbers may occur at random. For example, in some reliability experiments, an experimenter may decide that it is inappropriate or too dangerous to carry on the testing on some of the tested units even though these units have not failed. In such cases, the pattern of removal at each failure is random $[7,8]$. This leads to progressive censoring with random removals (illustrated by Table 1). There have been several references about the statistical inference on lifetime distributions under progressive censoring with random removals. Wu et al. [9] $\mathrm{Wu}$ and Chang [10] considered the estimation problem for the Burr type-XII distribution and Pareto distribution based on progressively censored samples with random removals, where the number of units removed at each failure time has a discrete uniform removal pattern and binomial distribution.

\section{Maximum Likelihood Estimation}

Let $X_{1}<X_{2}<\cdots<X_{m}$ be the ordered failure times out of $n$ randomly selected times, where $m$ is predetermined before the test. At the $i$ th failure, $R_{i}$ items are removed from the test. For progressive censoring with predetermined number of removals $R=\left(R_{1}=r_{1}, \ldots, R_{m-1}=r_{1}\right)$, the likelihood function can be defined as the following form:

$$
L(\sigma, x \mid R)=c(r) \prod_{i=1}^{m} f\left(x_{i} \mid \sigma\right)\left[1-F\left(x_{i} \mid \sigma\right)\right]^{r_{i}}
$$

where $c(r)=n\left(n-1-r_{1}\right) \cdots\left(n-\sum_{i=1}^{m-1}\left(r_{i}+1\right)\right)$. Equation (4) is derived conditional on $r_{i}$. Each $r_{i}$ can be of any integer value between 0 and $n-m-\sum_{i=1}^{i-1}\left(r_{j}\right)$. It is different from progressive censoring with fixed removal that $r_{i}$ is a random number and is assumed to follow a binomial distribution with parameter $p$. It means that each unit leaves with equal probability $p$ and the probability of $r_{i}$ units leaving after the $i$ th failure occurs is

$$
\begin{gathered}
P\left(R_{1}=r_{1}\right)=\left(\begin{array}{c}
n-m \\
r_{1}
\end{array}\right) p^{r_{1}}(1-p)^{n-m-r_{1}}, \\
P\left(R_{i}=r_{i} \mid R_{i-1}=r_{i-1}, \ldots, R_{1}=r_{1}\right) \\
=\left(\begin{array}{c}
n-m-\sum_{j=1}^{i-1} r_{j} \\
r_{i}
\end{array}\right) p^{r_{i}}(1-p)^{n-m-\sum_{j=1}^{m-1} r_{j}},
\end{gathered}
$$

where $0 \leq r_{i} \leq n-m-\sum_{j=1}^{i-1} r_{j}(i=1, \ldots, m-1)$. Furthermore, we assume that $R_{i}$ is independent of $X_{i}$ for all $i$. The joint likelihood function of $X=\left(X_{1}, X_{2}, \ldots, X_{m}\right)$ and $R=\left(R_{1}, R_{2}, \ldots, R_{m}\right)$ can be found as

$$
L(\sigma, x, p)=L(\sigma, x \mid R) P(R, p),
$$

where $P(R, p)$ is the joint probability distribution of $R=$ $\left(r_{1}, r_{2}, \ldots, r_{m}\right)$ and in particular

$$
\begin{aligned}
P(R, p)= & P\left(R_{m}=r_{m} \mid R_{m-1}=r_{m-1}, \ldots, R_{1}=r_{1}\right) \\
& \times P\left(R_{2}=r_{2} \mid R_{1}=r_{1}\right) P\left(R_{1}=r_{1}\right) .
\end{aligned}
$$

Therefore

$$
\begin{aligned}
P(R, p)= & \frac{(n-m) !}{\left(n-m-\sum_{j=1}^{m-1} r_{j}\right) ! \prod_{j=1}^{m-1} r_{j}} p^{\sum_{j=1}^{m-1} r_{j}} \\
& \times(1-p)^{(m-1)(n-m)-\sum_{j=1}^{m-1}(m-j) r_{j}} .
\end{aligned}
$$

Substituting (1) and (2) into (6), the likelihood function takes the following form:

$$
\begin{aligned}
L(\sigma, x, p) \propto & \sigma^{-2 m} \exp \left\{-\frac{1}{2 \sigma^{2}} \sum_{i=1}^{m}\left(R_{i}+1\right) x_{i}^{2}\right\} p^{\sum_{j=1}^{m-1} r_{j}} \\
& \times(1-p)^{(m-1)(n-m)-\sum_{j=1}^{m-1}(m-j) r_{j}} .
\end{aligned}
$$

Thus, the log-likelihood function takes the following form:

$$
\begin{aligned}
l=\ln L= & -2 m \ln \sigma-\frac{1}{2 \sigma^{2}} \sum_{i=1}^{m}\left(R_{i}+1\right) x_{i}^{2}+\left(\sum_{j=1}^{m-1} r_{j}\right) \ln P \\
& +\left((m-1)(n-m)-\sum_{j=1}^{m-1}(m-j) r_{j}\right) \ln (1-P) .
\end{aligned}
$$

The first partial derivatives of log-likelihood function $l$ with respect to $\sigma$ and $p$ are

$$
\begin{gathered}
\frac{\partial l}{\partial \sigma}=-\frac{2 m}{\sigma}+\frac{\sum_{i=1}^{m}\left(r_{i}+1\right) x_{i}^{2}}{\sigma^{3}}=0 \\
\frac{\partial l}{\partial P}=\frac{\sum_{j=1}^{m-1} r_{j}}{P}-\frac{(m-1)(n-m)-\sum_{j=1}^{m-1}(m-j) r_{j}}{1-P}=0 .
\end{gathered}
$$


Solving these equations in $\sigma$ and $p$, we get the MLE of $\sigma$ and $p$ as in the following forms:

$$
\begin{gathered}
\widehat{\sigma}=\sqrt{\frac{\sum_{i=1}^{m}\left(r_{i}+1\right) x_{i}^{2}}{2 m}}, \\
\widehat{p}=\frac{\sum_{j=1}^{m-1} r_{j}}{(m-1)(n-m)-\sum_{j=1}^{m-1}(m-j) r_{j}+\sum_{j=1}^{m-1} r_{j}} .
\end{gathered}
$$

It is clear that the MLE of reliability function, $R\left(x_{0}\right)$, with fixed $x_{0}>0$, can be obtained by

$$
\widehat{R}=\exp \left\{-\frac{x_{0}^{2}}{2 \widehat{\sigma}^{2}}\right\}
$$

\section{Confidence Intervals for $\sigma$}

Let $Y_{i}=X_{i}^{2} / 2 \sigma^{2}, i=1, \ldots, m$. It is easy to show that $Y_{1}, \ldots, Y_{m}$ is a progressively type II censored sample from the standard exponential distribution. For a fixed set of $R=$ $\left(r_{1}, \ldots, r_{m}\right)$, let us consider the following transformation:

$$
\begin{aligned}
& Z_{1}=n Y_{1}, \\
& Z_{2}=\left(n-r_{1}-1\right)\left(Y_{2}-Y_{1}\right), \\
& \vdots \\
& Z_{m}=\left(n-r_{1}-\cdots-r_{m}-m+1\right)\left(Y_{m}-Y_{m-1}\right) .
\end{aligned}
$$

Thomas and Wilson [11] showed that the generalized spacings $Z_{1}, \ldots, Z_{m}$, as defined in (14), are all independent and identically distributed as standard exponential. Hence, $2 Z_{1}$ has a Chi-square distribution with 2 degrees of freedom. We can also write the numerator of $\widehat{\sigma}^{2}$ as the sum of $m$-independent generalized spacings. That is, $2 \sum_{i=1}^{m} Z_{i}=$ $\sum_{i=1}^{m}\left(\left(r_{i}+1\right) X_{i}^{2} / \sigma^{2}\right)$. Therefore, we can find that, conditionally on a fixed set of $R=\left(r_{1}, \ldots, r_{m}\right), 2 m \widehat{\sigma}^{2} / \sigma^{2}$ has a Chi-square distribution with $2 m$ degrees of freedom.

Confidence interval for $\sigma^{2}$ can be obtained through the pivotal quantity $2 m \widehat{\sigma}^{2} / \sigma^{2} \sim \chi_{(2 m)}^{2}$ and can be the percentile of Chi-square distribution with $2 m$ degrees of freedom and right-tail probability $\alpha$. Then

$$
\left(\frac{2 m \widehat{\sigma}^{2}}{\chi_{1-(\alpha / 2)(2 m)}^{2}}, \frac{2 m \widehat{\sigma}^{2}}{\chi_{(\alpha / 2)(2 m)}^{2}}\right)
$$

since $\sigma^{2}$ is increasing function of $\sigma$. Then confidence interval for $\sigma$ is

$$
\left(\sqrt{\frac{2 m \widehat{\sigma}^{2}}{\chi_{1-(\alpha / 2)(2 m)}^{2}}}, \sqrt{\frac{2 m \widehat{\sigma}^{2}}{\chi_{(\alpha / 2)(2 m)}^{2}}}\right) .
$$

\section{Bayesian Estimation}

In this section, we use the Bayes procedure to derive the point estimates of the parameters $\sigma$ and $p$ and reliability function $R\left(x_{0}\right)$ based on progressively type II censoring data with binomial removals. For this purpose we assume the parameters $\sigma$ and $p$ behave as independent random variables. In this paper, for parameter $\sigma$ we consider prior distribution of the form

$$
\pi_{1}(\sigma)=\frac{a^{b}}{\Gamma(b) 2^{b-1}} \sigma^{-2 b-1} \exp \left\{-\frac{a}{2 \sigma^{2}}\right\}
$$

where $a>0$ and $b>0$. This density is known as the square-root inverted-gamma distribution (see [12, page 119]) and, independently from parameter $\sigma, p$ has a beta prior distribution with parameters $\alpha$ and $\beta$ and PDF

$$
\pi_{2}(p)=\frac{1}{B(\alpha, \beta)} p^{\alpha-1}(1-p)^{\beta-1}, \quad 0<p<1 ; \alpha, \beta>0
$$

Based on the prior $\pi_{1}(\sigma)$ and $\pi_{2}(p)$, the joint prior PDF of $(\sigma, p)$ is

$$
\begin{aligned}
\pi(\sigma, p)= & \pi_{1}(\sigma) \pi_{2}(p), \quad \sigma>0,0<p<1 \\
= & \frac{a^{b}}{B(\alpha, \beta) \Gamma(b) 2^{b-1}} p^{\alpha-1} \\
& \times(1-p)^{\beta-1} \sigma^{-2 b-1} \exp \left\{-\frac{a}{2 \sigma^{2}}\right\}
\end{aligned}
$$

It follows, from (9) and (20), that the joint posterior distribution of $(\sigma, p)$ is given by

$$
\begin{aligned}
\pi(\sigma, p \mid \mathbf{x}, \mathbf{r})= & \frac{a^{* b^{*}}}{B\left(\alpha^{*}, \beta^{*}\right) \Gamma\left(b^{*}\right) 2^{b^{*}-1}} p^{\alpha^{*}-1} \\
& \times(1-p)^{\beta^{*}-1} \sigma^{-2 b^{*}-1} \exp \left\{-\frac{a^{*}}{2 \sigma^{2}}\right\},
\end{aligned}
$$

where $a^{*}=a+\sum_{i=1}^{m}\left(R_{i}+1\right) x_{i}^{2}, b^{*}=m+b, \alpha^{*}=\alpha+$ $\sum_{j=1}^{m-1} r_{j}, \beta^{*}=\beta+(m-1)(n-m)-\sum_{j=1}^{m-1}(m-j) r_{j}$.

Therefore, the marginal posterior PDFs of $\sigma$ and $p$ are given, respectively, by

$$
\begin{array}{r}
\pi(\sigma \mid \mathbf{x}, \mathbf{r})=\frac{a^{* b^{*}}}{\Gamma\left(b^{*}\right) 2^{b^{*}-1}} \sigma^{-2 b^{*}-1} \exp \left\{-\frac{a^{*}}{2 \sigma^{2}}\right\}, \\
a^{*}>0, b^{*}>0, \\
\pi(p \mid \mathbf{x}, \mathbf{r})=\frac{1}{B\left(\alpha^{*}, \beta^{*}\right)} p^{\alpha^{*}-1}(1-p)^{\beta^{*}-1}, \\
0<p<1 ; \alpha^{*}, \beta^{*}>0 .
\end{array}
$$


Substituting $\sigma^{2}=-x_{0}^{2} / 2 \log s$ into (22), we obtain the posterior probability density function of $s=R\left(x_{0} \mid \sigma\right)$ as

$$
\pi(s \mid \mathbf{x}, \mathbf{r})=\frac{1}{\Gamma\left(b^{*}\right)}\left(\frac{a^{*}}{x_{0}^{2}}\right)^{b^{*}}(-\log s)^{b^{*}-1} s^{a^{*} / x_{0}^{2}-1}
$$

for $0<s<1$.

4.1. Bayesian Estimation under Squared Error Loss Function. Under squared error loss, the Bayes estimator of $\sigma$ is the mean of posterior density (22), which is given by

$$
\widehat{\sigma}_{S}=\sqrt{\frac{a^{*}}{2}} \frac{\Gamma\left(b^{*}-1 / 2\right)}{\Gamma\left(b^{*}\right)} .
$$

And for parameter $p$, the Bayes estimator of $p$ is

$$
\widehat{p}_{S}=\frac{\alpha^{*}}{\alpha^{*}+\beta^{*}} .
$$

Finally, the Bayes estimators of $R\left(x_{0}\right)$ are found to be

$$
\widehat{R}_{S}=\left(\frac{a^{*}}{a^{*}+x_{0}^{2}}\right)^{b^{*}} \text {. }
$$

4.2. Bayesian Estimation under Entropy Loss Function. In many practical situations, it appears to be more realistic to express the loss in terms of the ratio $\hat{\theta} / \theta, \theta=(\sigma, p)$. In this case, Calabria and Pulcini [13] point out that a useful asymmetric loss function is the entropy loss function:

$$
L(\delta) \propto\left[\delta^{d}-d \log _{e}(\delta)-1\right]
$$

where

$$
\delta=\frac{\widehat{\theta}}{\theta}
$$

whose minimum occurs at $\widehat{\theta}=\theta$. The latter has been used in Dey et al., [14] and Dey and Liu [15], in the original form having $d=1$ :

$$
L(\delta)=b\left[\delta-\log _{e}(\delta)-1\right], \quad b>0 .
$$

Therefore, the Bayesian estimators of parameters $\theta=(\sigma, p)$ under the entropy loss function with $d=1$, denoted by $\widehat{\theta}_{E}$, is given by the following equation:

$$
\widehat{\theta}_{E}=\left[E\left(\frac{1}{\theta} \mid \mathbf{x}, \mathbf{r}\right)\right]^{-1} \text {. }
$$

Therefore we have

$$
\begin{gathered}
\widehat{\sigma}_{E}=\sqrt{\frac{a^{*}}{2}} \frac{\Gamma\left(b^{*}\right)}{\Gamma\left(b^{*}+1 / 2\right)}, \\
\widehat{p}_{E}=\frac{\alpha^{*}-1}{\alpha^{*}+\beta^{*}-1}, \\
\widehat{R}_{E}=\left(1-\frac{x_{0}^{2}}{a^{*}}\right)^{b^{*}} .
\end{gathered}
$$

4.3. Bayesian Estimation under Precautionary Loss Function. Precautionary loss function which is asymmetric, was introduced by Norström [16]:

$$
L(\widehat{\theta}, \theta)=\frac{(\widehat{\theta}-\theta)^{2}}{\widehat{\theta}} .
$$

The Bayes estimator under the latter loss function is denoted by $\widehat{\theta}_{P}$ and may be obtained by solving the following equation:

$$
\widehat{\theta}_{P}^{2}=E\left(\theta^{2} \mid \mathbf{x}, \mathbf{r}\right) \text {. }
$$

The Bayesian estimators of the parameters $\sigma, p$ and $R(t)$ under asymmetric precautionary loss function are given, respectively, by

$$
\begin{gathered}
\widehat{\sigma}_{P}=\sqrt{\frac{a^{*}}{2\left(b^{*}-1\right)},} \\
\widehat{p}_{P}=\left(\frac{\alpha^{*}\left(\alpha^{*}+1\right)}{\left(\alpha^{*}+\beta^{*}+1\right)\left(\alpha^{*}+\beta^{*}\right)}\right)^{1 / 2}, \\
\widehat{R}_{P}=\left(\frac{a^{*}}{a^{*}+2 x_{0}^{2}}\right)^{b^{*} / 2} .
\end{gathered}
$$

\section{Numerical Study}

In our study, we firstly generate the numbers of progressive censoring with binomial removals $r_{i}(i=1,2, \ldots, m)$, and then we get the progressive censoring with binomial removals samples from Rayleigh distribution by the Monte-Carlo method. The steps are as follows.

Step 1. Generate a group values

$$
\begin{gathered}
r_{i} \sim B\left(n-m-\sum_{j=1}^{i-1} r_{j}, p\right), \\
r_{m}=n-m-\sum_{i=1}^{m-1} r_{i}, \quad i=1,2, \ldots, m-1
\end{gathered}
$$

according to the relevant value of $p$.

Step 2. Simulate $m$-independent exponential random variables $Z_{1}, Z_{2}, \ldots, Z_{m}$.

This can be done using inverse transformation $Z_{i}=$ $-\ln \left(1-U_{i}\right)$, where $U_{i}$ are independent uniform $(0,1)$ random variables.

Step 3. Set

$$
\begin{aligned}
X_{i}= & \frac{Z_{1}}{n}+\frac{Z_{2}}{n-R_{1}-1}+\frac{Z_{3}}{n-R_{1}-R_{2}-2}+\cdots \\
& +\frac{Z_{i}}{n-R_{1}-R_{2}-\cdots-R_{i-1}-i+1}
\end{aligned}
$$

for $i=1,2, \ldots, m$. This is the required progressively type II censored sample with binomial removals from the standard exponential distribution. 
TABLE 2: Averaged values of MSEs for estimates of the parameters $\theta$ and $p$.

\begin{tabular}{|c|c|c|c|c|c|c|c|c|c|c|c|}
\hline & $n$ & $m$ & $\widehat{\sigma} \mathrm{MSE}$ & $\widehat{\sigma}_{S}$ MSE & $\widehat{\sigma}_{E} \mathrm{MSE}$ & $\widehat{\sigma}_{P} \mathrm{MSE}$ & $\mathrm{CI}$ & $\widehat{p}$ MSE & $\widehat{p}_{S}$ MSE & $\widehat{p}_{E}$ MSE & $\widehat{p}_{P} \mathrm{MSE}$ \\
\hline \multirow{8}{*}{$P=0.1$} & \multirow{4}{*}{10} & \multirow{2}{*}{5} & 0.9727 & 1.0484 & 1.0749 & 1.0019 & \multirow{2}{*}{$(0.6796,1.7070)$} & 0.1333 & 0.2500 & 0.2105 & 0.1336 \\
\hline & & & 0.0514 & 0.0416 & 0.0469 & 0.0359 & & 0.0011 & 0.0225 & 0.0122 & 0.0011 \\
\hline & & \multirow{2}{*}{7} & 0.9794 & 1.0334 & 1.0520 & 0.9996 & \multirow{2}{*}{$(0.7170,1.5447)$} & 0.0769 & 0.2222 & 0.1764 & 0.1139 \\
\hline & & & 0.0343 & 0.0292 & 0.0319 & 0.0263 & & 0.0005 & 0.0149 & 0.0058 & 0.0001 \\
\hline & \multirow{4}{*}{20} & \multirow{2}{*}{10} & 0.9873 & 1.0250 & 1.0379 & 1.0009 & \multirow{2}{*}{$(0.7554,1.4258)$} & 0.1228 & 0.1612 & 0.1475 & 0.0673 \\
\hline & & & 0.0251 & 0.0226 & 0.0239 & 0.0209 & & 0.0005 & 0.0037 & 0.0022 & 0.0010 \\
\hline & & \multirow{2}{*}{15} & 0.9889 & 1.0141 & 1.0226 & 0.9979 & \multirow{2}{*}{$(0.7902,1.3218)$} & 0.0975 & 0.1521 & 0.1333 & 0.0627 \\
\hline & & & 0.0165 & 0.0153 & 0.0158 & 0.0146 & & $5 \times 10^{-6}$ & 0.0027 & 0.0011 & 0.0013 \\
\hline \multirow{8}{*}{$P=0.4$} & \multirow{4}{*}{10} & \multirow{2}{*}{5} & 0.9700 & 1.0461 & 1.0726 & 0.9997 & \multirow{2}{*}{$(0.6778,1.7024)$} & 0.5000 & 0.5333 & 0.5000 & 0.4000 \\
\hline & & & 0.0522 & 0.0419 & 0.0471 & 0.0363 & & 0.01000 & 0.0177 & 0.0100 & 0.0000 \\
\hline & & \multirow{2}{*}{7} & 0.9805 & 1.0345 & 1.0532 & 1.0006 & \multirow{2}{*}{$(0.7178,1.5463)$} & 0.2500 & 0.3846 & 0.3333 & 0.2517 \\
\hline & & & 0.0371 & 0.0318 & 0.0346 & 0.0287 & & 0.0225 & 0.0002 & 0.0044 & 0.0219 \\
\hline & \multirow{4}{*}{20} & \multirow{2}{*}{10} & 0.9842 & 1.0221 & 1.0349 & 0.9980 & \multirow{2}{*}{$(0.7530,1.4213)$} & 0.3703 & 0.4062 & 0.3870 & 0.2646 \\
\hline & & & 0.0243 & 0.0216 & 0.0229 & 0.0202 & & 0.0008 & 0.0009 & 0.0001 & 0.0183 \\
\hline & & \multirow{2}{*}{15} & 0.9897 & 1.0149 & 1.0234 & 0.9986 & \multirow{2}{*}{$(0.7909,1.3230)$} & 0.4545 & 0.5000 & 0.4666 & 0.3638 \\
\hline & & & 0.0163 & 0.0151 & 0.0157 & 0.0144 & & 0.0029 & 0.0100 & 0.0044 & 0.0013 \\
\hline \multirow{8}{*}{$P=0.6$} & \multirow{4}{*}{10} & \multirow{2}{*}{5} & 0.9706 & 1.0466 & 1.0730 & 1.0001 & \multirow{2}{*}{$(0.6782,1.7034)$} & 0.7142 & 0.6666 & 0.6363 & 0.5547 \\
\hline & & & 0.0511 & 0.0411 & 0.0462 & 0.03557 & & 0.0130 & 0.0044 & 0.0013 & 0.0020 \\
\hline & & \multirow{2}{*}{7} & 0.9818 & 1.0356 & 1.0542 & 1.0017 & $(0.7188,1.5485)$ & 0.2727 & 0.3750 & 0.3333 & 0.2406 \\
\hline & & & 0.0343 & 0.0296 & 0.0323 & 0.0265 & & 0.1071 & 0.0506 & 0.0711 & 0.1291 \\
\hline & & 10 & 0.9906 & 1.0280 & 1.0409 & 1.0038 & & 0.6666 & 0.6500 & 0.6315 & 0.5307 \\
\hline & 20 & 10 & 0.0240 & 0.0219 & 0.0233 & 0.0201 & $(0 . / 5 / 9,1.4505)$ & 0.0044 & 0.0025 & 0.0009 & 0.0047 \\
\hline & & 15 & 0.9913 & 1.0164 & 1.0249 & 1.0001 & $(0.7922,1.3251)$ & 0.4545 & 0.5000 & 0.4666 & 0.3638 \\
\hline & & & 0.0161 & 0.0150 & 0.0156 & 0.0143 & (0.0. & 0.0211 & 0.0100 & 0.0177 & 0.0557 \\
\hline & & 5 & 0.9746 & 1.0500 & 1.0766 & 1.0034 & & 0.7142 & 0.6666 & 0.6363 & 0.5547 \\
\hline & 10 & & 0.0499 & 0.0408 & 0.0461 & 0.0349 & & 0.0344 & 0.0544 & 0.0695 & 0.1192 \\
\hline & & 7 & 0.9779 & 1.0322 & 1.0508 & 0.9984 & $(0.7159,1.5423)$ & 0.7500 & 0.6666 & 0.6250 & 0.5577 \\
\hline$P=0.9$ & & 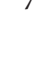 & 0.0370 & 0.0315 & 0.0341 & 0.0285 & $(0 . / 159,1.5423)$ & 0.0225 & 0.05447 & 0.0756 & 0.1171 \\
\hline$P=0.9$ & & 10 & 0.9882 & 1.0258 & 1.0387 & 1.0017 & $(07560,14270)$ & 0.9090 & 0.8125 & 0.8000 & 0.7373 \\
\hline & 20 & 10 & 0.0248 & 0.0223 & 0.0237 & 0.0207 & $(0.1560,1.42 / 0)$ & $8 \times 10^{-5}$ & 0.0076 & 0.0100 & 0.0264 \\
\hline & & 15 & 0.9931 & 1.0181 & 1.0266 & 1.0018 & & 0.6250 & 0.6153 & 0.5833 & 0.4934 \\
\hline & & & 0.0153 & 0.0143 & 0.0149 & 0.0136 & & 0.0756 & 0.0810 & 0.1002 & 0.1653 \\
\hline
\end{tabular}

Step 4. Finally, we set $Y_{i}=F^{-1}\left(1-\exp \left(-X_{i}\right)\right)$, for $i=1,2, \ldots, m$, where $F^{-1}(\cdot)$ is the inverse cumulative distribution function of the Rayleigh distribution. Then $Y_{1}, Y_{2}, \ldots, Y_{m}$ is the required progressively type II censored sample with binomial removals from the Rayleigh distribution.

Step 5. We compute the MLE of parameters $\sigma$ and $p$ by (12), for reliability function by (13) and 95\% confidence intervals by (16).

Step 6. We compute the the Bayes estimates of parameters and reliability function, respectively, using (25), (32), (37), (26), (33), (38), (27), (34), and (39).
Step 7. We repeat the above steps 2000 times. We then obtain the means and the MSEs (mean-squared error) for different censoring sizes $m$ and censoring schemes where

$$
\mathrm{MSE}=2000^{-1} \sum_{i=1}^{2000}\left(\phi-\widehat{\phi}_{i}\right)^{2}
$$

and $\widehat{\phi}$ is the estimator of $\phi$.

In all above cases the prior parameters chosen as $(a=2$, $b=1)$ and $(\alpha=3, \beta=2)$ which yield the generated value of $\sigma=1$ as the true value. The true values of $R\left(x_{0}\right)$ in $x_{0}=0.5$ is obtained $R(0.5)=0.8824969$. The results are summarized in Tables 2 and 3. 
TABLE 3: Averaged values of MSEs for estimates of the reliability function.

\begin{tabular}{|c|c|c|c|c|c|c|}
\hline & $n$ & $m$ & $\widehat{R}$ MSE & $\widehat{R}_{S}$ MSE & $\widehat{R}_{E}$ MSE & $\widehat{R}_{P}$ MSE \\
\hline \multirow{8}{*}{$P=0.1$} & \multirow{4}{*}{10} & \multirow{2}{*}{5} & 0.8578 & 0.8353 & 0.8297 & 0.8379 \\
\hline & & & 0.0052 & 0.0078 & 0.0094 & 0.0072 \\
\hline & & \multirow{2}{*}{7} & 0.8662 & 0.8505 & 0.8472 & 0.8520 \\
\hline & & & 0.0030 & 0.0042 & 0.0048 & 0.0040 \\
\hline & \multirow{4}{*}{20} & \multirow{2}{*}{10} & 0.8714 & 0.8606 & 0.8586 & 0.8615 \\
\hline & & & 0.0018 & 0.0024 & 0.0026 & 0.0023 \\
\hline & & \multirow{2}{*}{15} & 0.8764 & 0.8694 & 0.8682 & 0.8699 \\
\hline & & & 0.0010 & 0.0012 & 0.0013 & 0.0012 \\
\hline \multirow{8}{*}{$P=0.4$} & \multirow{4}{*}{10} & \multirow{2}{*}{5} & 0.8589 & 0.8366 & 0.8310 & 0.8392 \\
\hline & & & 0.0053 & 0.0079 & 0.0094 & 0.0072 \\
\hline & & \multirow{2}{*}{7} & 0.8660 & 0.8503 & 0.8470 & 0.8519 \\
\hline & & & 0.0030 & 0.0042 & 0.0048 & 0.0040 \\
\hline & \multirow{4}{*}{20} & \multirow{2}{*}{10} & 0.8690 & 0.8581 & 0.8560 & 0.8590 \\
\hline & & & 0.0020 & 0.0026 & 0.0029 & 0.0025 \\
\hline & & \multirow{2}{*}{15} & 0.8744 & 0.8673 & 0.8661 & 0.8679 \\
\hline & & & 0.0010 & 0.0013 & 0.0014 & 0.0012 \\
\hline \multirow{8}{*}{$P=0.6$} & \multirow{4}{*}{10} & \multirow{2}{*}{5} & 0.8592 & 0.8369 & 0.8313 & 0.8394 \\
\hline & & & 0.0052 & 0.0078 & 0.0092 & 0.0071 \\
\hline & & \multirow{2}{*}{7} & 0.8672 & 0.8516 & 0.8484 & 0.8532 \\
\hline & & & 0.0030 & 0.0042 & 0.0047 & 0.0040 \\
\hline & \multirow{4}{*}{20} & \multirow{2}{*}{10} & 0.8710 & 0.8601 & 0.8582 & 0.8611 \\
\hline & & & 0.0018 & 0.0024 & 0.0026 & 0.0023 \\
\hline & & \multirow{2}{*}{15} & 0.8749 & 0.8678 & 0.8666 & 0.8684 \\
\hline & & & 0.0010 & 0.0013 & 0.0014 & 0.0012 \\
\hline \multirow{8}{*}{$P=0.9$} & \multirow{4}{*}{10} & \multirow{2}{*}{5} & 0.8593 & 0.8370 & 0.8315 & 0.8396 \\
\hline & & & 0.0052 & 0.0077 & 0.0092 & 0.0071 \\
\hline & & \multirow{2}{*}{7} & 0.8660 & 0.8503 & 0.8470 & 0.8518 \\
\hline & & & 0.0032 & 0.0045 & 0.0051 & 0.0043 \\
\hline & \multirow{4}{*}{20} & \multirow{2}{*}{10} & 0.8725 & 0.8617 & 0.8598 & 0.8626 \\
\hline & & & 0.0016 & 0.0022 & 0.0024 & 0.0021 \\
\hline & & \multirow{2}{*}{15} & 0.8766 & 0.8695 & 0.8684 & 0.8700 \\
\hline & & & 0.0009 & 0.0011 & 0.0012 & 0.0011 \\
\hline
\end{tabular}

\section{Conclusions}

We develop some results on Rayleigh distribution when progressive type II censoring with binomial removals is performed. Based on the results shown in Table 2, one can conclude that Bayesian estimators under precautionary loss function have the smallest biased and MSE as compared with the other estimators. The MSE associated with the estimators (Bayesian estimators under different loss functions and maximum likelihood estimators) of the parameter $\sigma$ decrease with increasing the sample size $n$. According to Table 3 maximum likelihood estimators of reliability function of Rayleigh distribution have smallest biased and MSE as compared with the other estimators. It is immediate to note that MSEs of reliability function estimators decrease as $n, m$ increases.

\section{References}

[1] H. L. Harter and A. H. Moore, "Point and interval estimators, based on $\mathrm{m}$ order statistics, for the scale parameter of a Weibull population with known shape parameter," Technometrics, vol. 7, no. 3, pp. 405-422, 1995.

[2] A. J. Fernández, "Bayesian inference from type II doubly censored Rayleigh data," Statistics and Probability Letters, vol. 48, no. 4, pp. 393-399, 2000.

[3] M. Z. Raqab and M. T. Madi, "Bayesian prediction of the total time on test using doubly censored Rayleigh data," Journal of Statistical Computation and Simulation, vol. 72, no. 10, pp. 781789, 2002.

[4] S. J. Wu, D. H. Chen, and S. T. Chen, "Bayesian inference for Rayleigh distribution under progressive censored sample," Applied Stochastic Models in Business and Industry, vol. 22, no. 3, pp. 269-279, 2006.

[5] N. Balakrishnan and R. Aggarwala, Progressive Censoring: Theory, Methods, and Applications, Birkhäauser, Boston, Mass, USA, 2000.

[6] N. Balakrishnan, "Progressive censoring methodology: an appraisal," Test, vol. 16, no. 2, pp. 290-296, 2007.

[7] Z. H. Amin, "Bayesian inference for the Pareto lifetime model under progressive censoring with binomial removals," Journal of Applied Statistics, vol. 35, no. 11, pp. 1203-1217, 2008.

[8] H. K. Yuen and S. K. Tse, "Parameters estimation for weibull distributed lifetimes under progressive censoring with random removals," Journal of Statistical Computation and Simulation, vol. 55, no. 1-2, pp. 57-71, 1996.

[9] S. J. Wu, Y. J. Chen, and C. T. Chang, "Statistical inference based on progressively censored samples with random removals from the Burr type XII distribution," Journal of Statistical Computation and Simulation, vol. 77, no. 1, pp. 19-27, 2007.

[10] S. J. Wu and C. T. Chang, "Inference in the Pareto distribution based on progressive type II censoring with random removals," Journal of Applied Statistics, vol. 30, no. 2, pp. 163-172, 2003.

[11] D. R. Thomas and W. M. Wilson, "Linear order statistic estimation for the two-parameter Weibull and extreme value distributions from type-II progressively censored samples," Technometrics, vol. 14, no. 3, pp. 679-691, 1972.

[12] J. M. Bernardo and A. F. M. Smith, Bayesian Theory, John Wiley \& Sons, New York, NY, USA, 1994.

[13] R. Calabria and G. Pulcini, "An engineering approach to Bayes estimation for the Weibull distribution," Microelectronics Reliability, vol. 34, no. 5, pp. 789-802, 1994.

[14] D. K. Dey, M. Ghosh, and C. Srinivasan, "Simultaneous estimation of parameters under entropy loss," Journal of Statistical Planning and Inference, vol. 15, pp. 347-363, 1986.

[15] D. K. Dey and P. S. L. Liu, "On comparison of estimators in a generalized life model," Microelectronics Reliability, vol. 32, no. 1-2, pp. 207-221, 1992.

[16] J. G. Norström, "The use of precautionary loss functions in risk analysis," IEEE Transactions on Reliability, vol. 45, no. 3, pp. 400-403, 1996. 

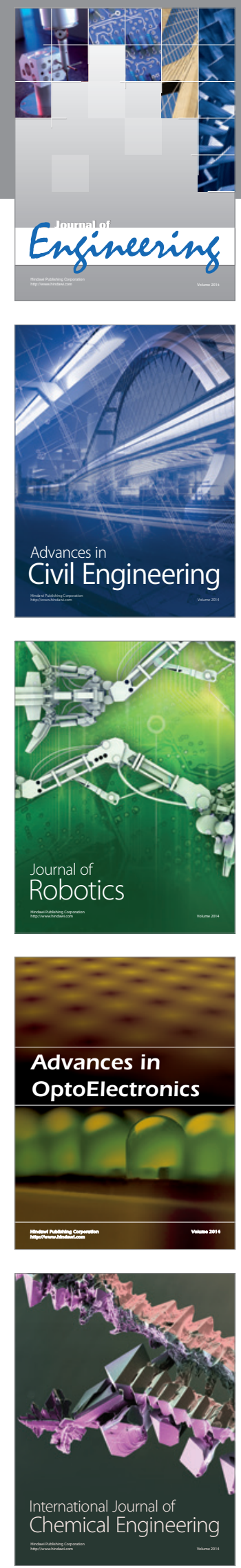

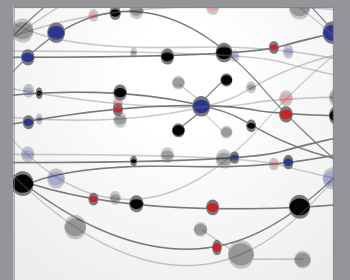

The Scientific World Journal
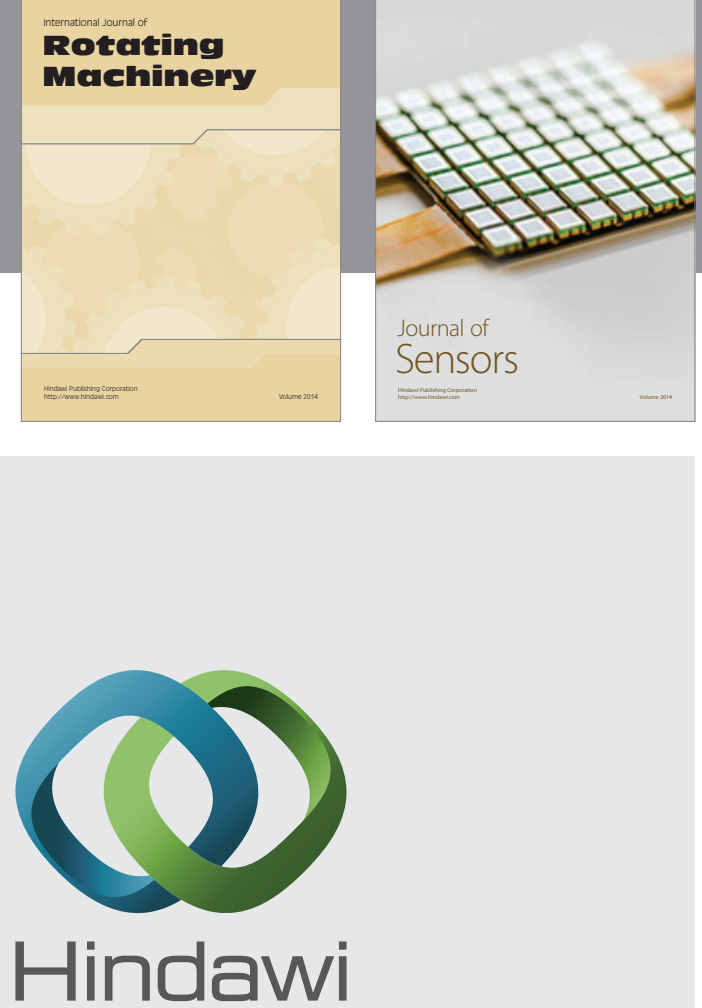

Submit your manuscripts at http://www.hindawi.com
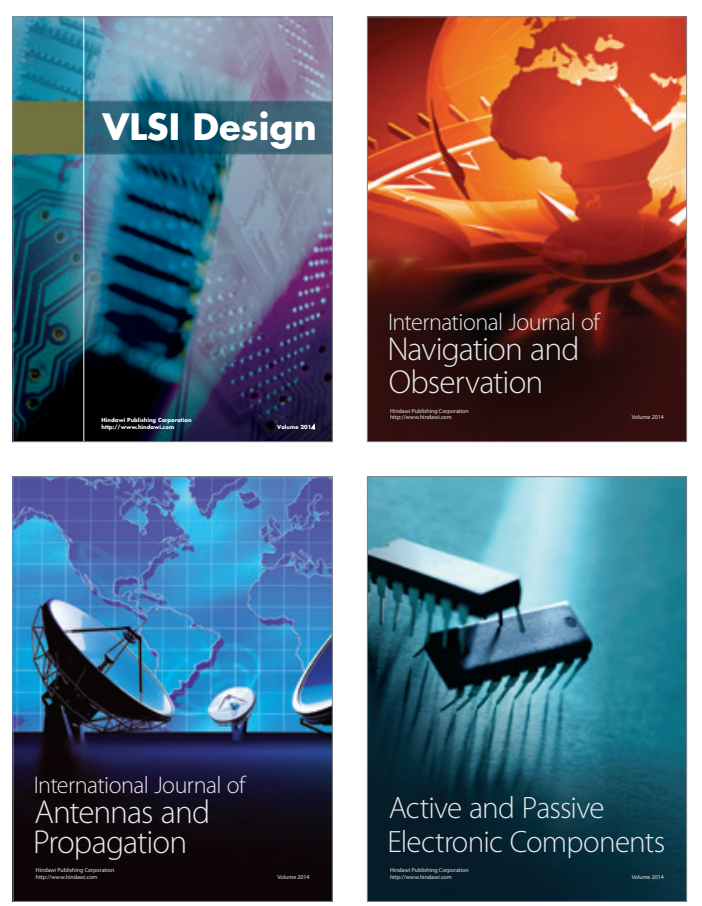
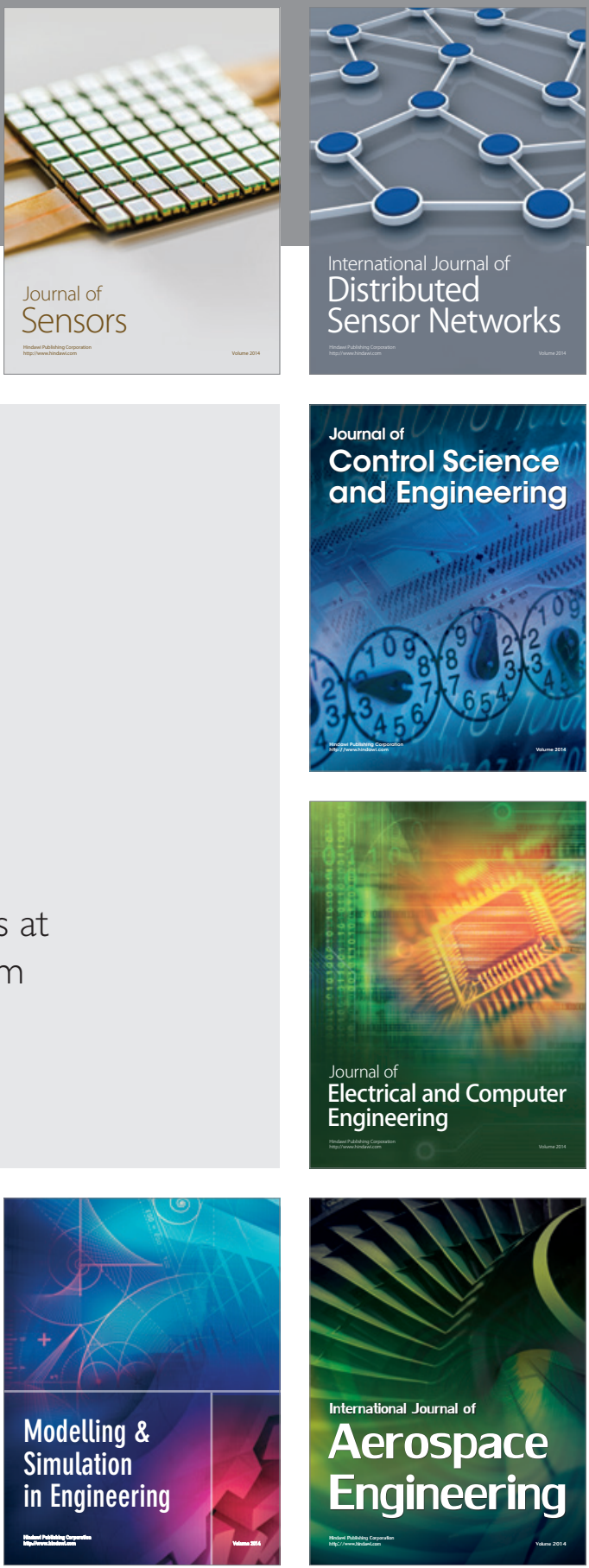

Journal of

Control Science

and Engineering
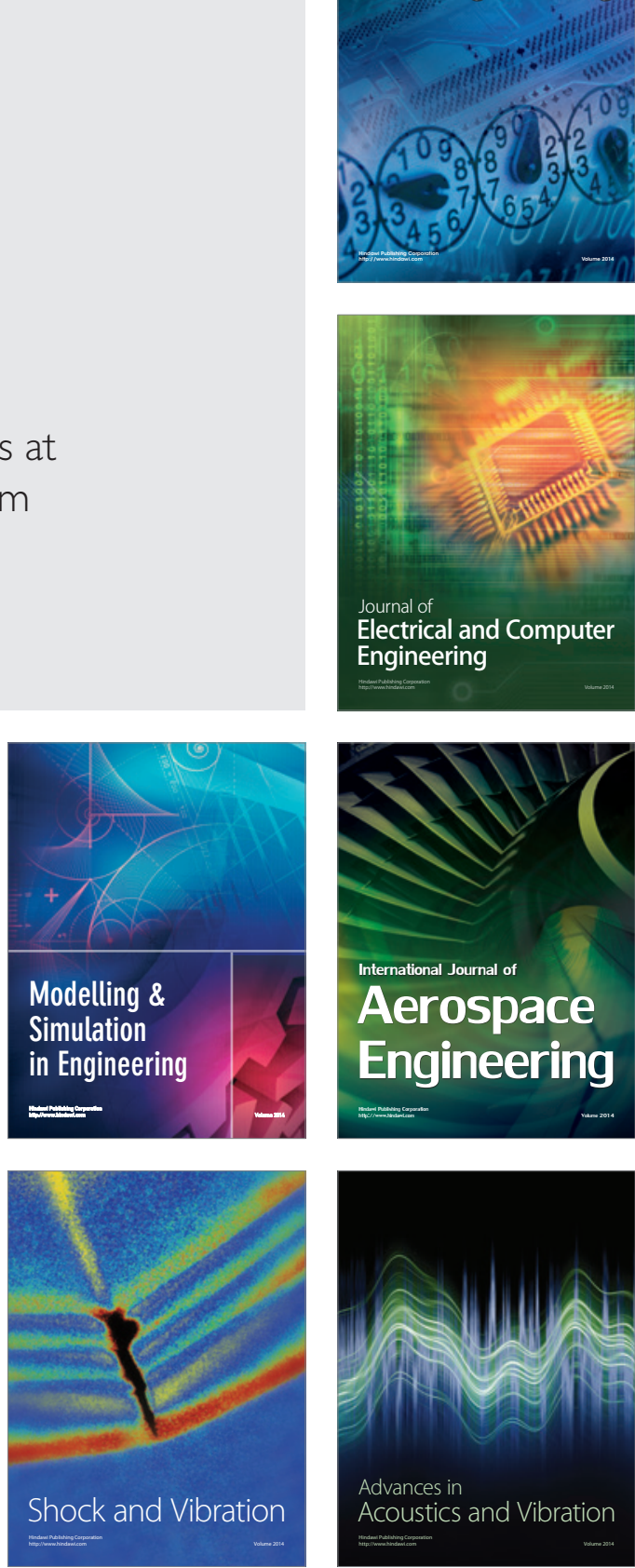